Rita de Cássia Silveira ${ }^{1}$, Clarice Giacomini², Renato Soibelmann Procianoy $^{3}$

1. Professor Adjunto do Departamento de Pediatria da Universidade Federal do Rio Grande do Sul - UFRGS - Porto Alegre (RS), Brasil.

2. Professor Assistente do Departamento de Pediatria da Universidade Federal do Rio Grande do Sul - UFRGS - Porto Alegre (RS), Brasil.

3. Professor Titular do Departamento de Pediatria da Universidade Federal do Rio Grande do Sul - UFRGS - Porto Alegre (RS), Brasil.

Recebido do Serviço de Neonatologia do Hospital de Clínicas de Porto Alegre - HCPA e do Departamento de Pediatria Universidade Federal do Rio Grande do Sul - UFRGS - Porto Alegre (RS), Brasil.

Submetido em 24 de Junho de 2010 Aceito em 5 de Agosto de 2010

Autor para correspondência: Rita de Cássia Silveira Rua Silva Jardim, 1155/701 CEP: 90450-071 - Porto Alegre (RS), Brasil.

E-mail: rita.c.s@terra.com.br

\section{Sepse e choque séptico no período neonatal: atualização e revisão de conceitos}

\author{
Neonatal sepsis and septic shock: concepts update and review
}

\section{RESUMO}

A sepse neonatal e a síndrome da resposta inflamatória sistêmica, que antecede o choque séptico, se manifestam como um estado não específico, o que pode retardar o diagnóstico precoce do choque séptico, razão pela qual a mortalidade desta condição permanece elevada. $\mathrm{O}$ diagnóstico precoce envolve a suspeita de choque séptico em todo recém nascido apresentando taquicardia, desconforto respiratório, dificuldade de alimentação, tônus alterado, cor alterada, taquipnéia e perfusão reduzida, especialmente na presença de histórico materno de infecção periparto, como corioamnionite ou ruptura prolongada de membranas ovulares.

O presente artigo tem como objetivo revisar o conhecimento atual a respeito das peculiaridades do período neonatal, da dinâmica da circulação fetal e da variável idade gestacional.

$\mathrm{O}$ choque séptico no recém-nascido não é choque séptico do adulto pequeno. No recém-nascido, o choque séptico é predominantemente frio, caracterizado por redução do débito cardíaco e alta resistência vascular sistêmica (vasoconstrição).

O tempo é fundamental no tratamento para reversão do choque séptico. A revisão da literatura, baseada em buscas em bases indexadas, fornece subsídios para o manejo do recém-nascido.

Descritores: Sepse; Recém-nascido; Choque séptico; Citocinas; Síndrome de resposta inflamatória sistêmica; Mortalidade neonatal

\section{INTRODUÇÃO}

Sepse neonatal com conseqüente choque séptico é causa de elevada morbidade e mortalidade infantil precoce há muitos anos. Sepse e o choque clássico por Streptococcus do grupo B permanecem sendo importante problema clínico a despeito de profilaxia antibiótica materna. ${ }^{(1)}$ De acordo com a Organização Mundial da Saúde (OMS), nascem em média 130 milhóes de recém-nascidos anualmente e destes, cerca de quatro milhóes morrem, sendo infecção a causa de 36\% desses óbitos e este problema torna-se mais relevante nos países onde a disponibilidade de recursos para o paciente crítico é limitada. ${ }^{(2)}$ Metade dos óbitos de recém-nascidos de muito baixo peso (RNMBP) após sete dias de vida, decorre de infecçóes graves. A infecção e a inflamação antenatal estão relacionadas ao trabalho de parto prematuro e ao desenvolvimento da displasia broncopulmonar. ${ }^{(3,4)}$ A exposição do cérebro prematuro a mediadores inflamatórios durante episódios infecciosos como coriamnionite, sepse e enterocolite está vinculado a hemorragia cerebral, lesão substância branca e alteraçóes do neurodesenvolvimento, inclusive paralisia cerebral. ${ }^{(3,5-9)}$ Doenças maternas e do feto (pré-eclampsia, restrição de crescimento intra-uterino) e do recém-nascido 
(persistência do canal arterial, e eventos micro-vasculares pósencefalopatia hipóxico- isquêmica) são cada vez mais delineadas pela ação de mediadores inflamatórios, o que pode estar relacionado ao reduzido número de sepse comprovada por hemocultura positiva no período neonatal imediato. ${ }^{(3,6)}$ Soma-se a estes fatos, um sistema imune ainda em desenvolvimento e que apresenta diferenças não só em relação a criança maior e ao adulto mas também entre os recém-nascidos, na dependência da idade gestacional. ${ }^{(9)}$

Apesar da resposta inflamatória sistêmica, caracterizada por taquicardia, taquipnéia, hipertermia, leucocitose ser mais frequentemente acionada pela presença de infecção, há diversos promotores da síndrome da resposta inflamatória sistêmica (SIRS) no período neonatal imediato, tais como: parto traumático, asfixia perinatal grave, erros inatos do metabolismo, procedimentos cirúrgicos, entre outras condiçóes que também são responsáveis pela liberação de mediadores inflamatórios, e estes orquestrarão a cascata de eventos próinflamatórios, que culmina no choque séptico. ${ }^{(10-15)}$

Uma força tarefa de intensivistas pediátricos da Critical Care Society coordenou recente atualizaçáo incorporando algumas novas recomendações graduadas em seu nível de evidência, sendo a definição de choque séptico baseada em parâmetros clínicos e o choque classificado conforme sua resposta à terapêutica instituída (Quadro 1). Muitas destas recomendaçôes não são obtidas a partir de estudos de boa qualidade e consistem em opinióes de consenso, mas a literatura demonstra queda nos índices de mortalidade com o uso destas diretrizes, sugerindo que o impacto maior é pela abordagem do atendimento, mais do que pelas intervençóes instituídas. ${ }^{(10)}$

Portanto, o objetivo consiste em revisar os principais estudos sobre o choque séptico no período neonatal, na busca do conhecimento das peculiaridades deste período, da dinâmica da circulação fetal e da variável idade gestacional (recémnascidos a termo, pré-termo e pré-termo extremo), o que será muito importante para o adequado manejo desta populaçáo de pacientes críticos.

\section{MÉTODOS}

Revisão da literatura, baseada em buscas em bases indexadas MEDLINE (PubMed) e Scientific Eletronic Library Online (SciELO). Foram revisados artigos originais com diferentes delineamentos, textos clássicos, cartas aos editores de periódicos indexados, meta-análises e suplementos de revisão com assuntos relacionados com choque séptico, sepse, bacteremia, fisiopatologia da sepse, resposta imuno-inflamatória dos recém-nascidos. A fonte de busca e o foco de interesse foi o período neonatal e suas particularidades. As palavraschaves incluíram: recém-nascido, pré-termo, choque, sepse, citocinas, resposta inflamatória, dopamina, dobutamina, noradrenalina, adrenalina, período de transição feto-neonatal.

$\mathrm{O}$ interesse em revisar especificamente choque séptico do recém-nascido, foi justificado pela maior freqüência em relação às demais causas de choque nesta fase da vida, pelas dificuldades de adequado tratamento, e em função de poucas evidências científicas quanto ao uso de inotrópicos no manejo do choque do recém-nascido.

Inicialmente, é estabelecida uma definição do choque séptico no recém-nascido. A apresentação da revisão da literatura particulariza o recém-nascido pré-termo, visto que pouco está estabelecido sobre diagnóstico e manejo do choque séptico no prematuro. Os Consensos e comitês internacionais estabeleceram critérios para disfunção multi-orgânica em recémnascidos mais novos (0-7 dias), e recém-nascidos maiores (7 dias a 1 mês), sem incluir na discussão os prematuros. ${ }^{(16,17)}$

Choque séptico deve ser classicamente definido pela presença de critérios diagnósticos de sepse, associado com disfunção cardiovascular sustentada, mesmo após infusão de, no mínimo, $40 \mathrm{ml} / \mathrm{kg}$ de volume em 1 hora. A disfunção cardiovascular causa, com freqüência, hipotensão (idade ges-

\section{Quadro 1- Definiçóes de choque séptico (de acordo com a força tarefa de intensivistas pediátricos da Critical Care Society)}

\begin{tabular}{|c|c|}
\hline Choque séptico (frio) & $\begin{array}{l}\text { Perfusão reduzida, incluindo estado mental alterado, enchimento capilar maior que } 2 \text { segundos, } \\
\text { pulso periférico reduzido, extremidades frias "moteadas" ou débito urinário reduzido (menor que } \\
1 \mathrm{ml} / \mathrm{kg} / \mathrm{h} \text { ). }\end{array}$ \\
\hline Choque séptico (quente) & $\begin{array}{l}\text { Perfusão reduzida, incluindo estado mental alterado, enchimento capilar menor que } 2 \text { segundos, } \\
\text { pulso periférico amplo, débito urinário reduzido (menor que } 1 \mathrm{ml} / \mathrm{kg} / \mathrm{h} \text { ). }\end{array}$ \\
\hline $\begin{array}{l}\text { Choque resistente à dopamina/ } \\
\text { refratário a líquidos: }\end{array}$ & $\begin{array}{l}\text { O choque persiste apesar de reanimação com líquidos }=60 \mathrm{ml} / \mathrm{kg} \text { na primeira hora e infusão de } \\
\text { dopamina a } 10 \mu \mathrm{g} / \mathrm{kg} / \mathrm{min} \text {. }\end{array}$ \\
\hline Choque resistente à catecolamina & O choque persiste apesar do uso de catecolamina adrenalina ou noradrenalina. \\
\hline Choque refratário & $\begin{array}{l}\text { O choque persiste apesar do uso direcionado de agentes inotrópicos, vasopressores, vasodilatado- } \\
\text { res e da manutenção da homeostase metabólica (glicose e cálcio) e hormonal (hormônio tireóideo } \\
\text { e hidrocortisona). }\end{array}$ \\
\hline
\end{tabular}

Modificado de Brierley J, Carcillo JA, Choong K, et al. ${ }^{(10)}$ 
tacional-dependente), necessidade de agentes vasopressores para manter pressão arterial ou evidência de perfusão periférica diminuída. ${ }^{(1,16,17)}$

$\mathrm{O}$ entendimento do choque no recém-nascido requer a descrição das variáveis, pressão arterial (PA) e débito cardíaco (Q). Existe uma relação básica entre PA, Q e resistência vascular periférica (RVP), bem conhecida e descrita pela fórmula:

$$
\text { Pressão Arterial = Q x RVP }
$$

Desta forma, uma pressão normal pode ocorrer com Q baixo se a RVP for alta.

No choque séptico neonatal, uma pressão arterial baixa irá ocorrer por baixo Q, pois a RVP costuma estar alta. Se o Q está normal e a RVP está alta, teremos hipertensão, o que pode ocorrer por várias razóes incluindo volume intravascular inadequado, pré-carga excessiva, contratilidade alterada, restrição miocárdica, disfunção valvular e arritmia. ${ }^{(17)}$

A apresentação desta revisão conta com subtítulos, dispostos após a introdução e a base conceitual da metodologia, de acordo com a proposta de revisóes sistemáticas de estudos clínicos randomizados realizados por comitês pediátricos. ${ }^{(18)}$ Inicialmente, é apresentado a fisiopatologia e o papel dos mediadores inflamatórios e do agente bacteriano; seguindo-se as diferenças entre o choque no recém-nascido e no paciente pediátrico, incluindo particularidades do choque no recémnascido, o choque e a pressão arterial sistêmica, e o manejo volumétrico e farmacológico (vasopressores e inotrópicos).

\section{Fisiopatologia: papel dos mediadores e do agente bac- teriano}

Diversas citocinas pró-inflamatórias são produzidas e secretadas a partir da presença do agente bacteriano na corrente sanguínea. Ao ativar neutrófilos e células endoteliais, estas interleucitocinas pró-inflamatórias possibilitam a adesão leucocitária na parede do endotélio, produção de óxido nítrico e outras espécies reativas de nitrogênio e oxigênio que irão causar maior dano endotelial com alteração das junçóes entre as células produzindo edema, vasodilatação e perda do controle do tônus vascular. A partir deste estado pró-inflamatório, poderá ocorrer progressão para um estado de imunossupressáo e imunoparalisia no compartimento sanguíneo, enquanto um estado hiperinflamatório tende a persistir em nível tecidual. ${ }^{(14,15,19)}$

Os recém-nascidos que apresentam quadro clínico de sepse grave sofrem todas as conseqüências do estado de hiperinflamação que leva ao choque séptico. Níveis de citocinas pro-inflamatórias como a IL-6, IL-1 $\beta$ e o TNF $\alpha$ estão significativamente mais elevados na presença de sepse neonatal precoce, constituindo-se em marcadores iniciais de infecção neonatal. ${ }^{(9,15)}$

Estudos que realizamos ainda na década passada, já demonstraram que a associação de IL-6 e TNF- $\alpha$ podem obter sensibilidade de $98,5 \%$, quando as citocinas são coletadas precocemente a partir do momento da suspeita de infecção. ${ }^{(14,20)}$ Nos prematuros extremos, estes níveis podem ser ainda mais elevados, especialmente IL-8, uma quimiocina que consiste em importante biomarcador de sepse neonatal. ${ }^{(21,22)}$ Por outro lado, a resposta compensatória antiinflamatória mediada por Toll-like receptors parece ser imatura até mesmo no recém-nascido de termo, um exemplo deste achado é a produção insuficiente de Interleucina-10 ( IL-10) e de TGF $\beta .^{(23-25)}$

OTNF- $\alpha$ é considerado o principal mediador do choque séptico e da lesão tecidual difusa, não apenas no recém-nascido, mas na criança e no adulto. ${ }^{(7,12,15)}$ Há diversas evidências que reforçam o papel do TNF- $\alpha$ na sepse, tais como: pacientes e modelos experimentais de choque séptico apresentam níveis máximos de TNF- $\alpha$, entre três e quatro horas do experimento; alteraçóes hemodinâmicas e metabólicas que provocam hipotensão, coagulação intravascular, necrose hemorrágica e lesão tecidual, mediadas pelo TNF- $\alpha$; a neutralização do TNF- $\alpha$ previne endotoxemia e bacteremia em modelos animais, mesmo quando endotoxinas e bactérias persistem na circulação sangüínea, porém esta terapêutica de neutralização do TNF- $\alpha$ não teve bons resultados no choque séptico. . $^{(7,15,20)}$

Durante o processo infeccioso o nível sérico de TNF- $\alpha$ eleva-se nos primeiros 30 a 90 minutos após a exposiçáo ao lipopolissacarídio (LPS) com pico entre 3 e 4 horas e tem boa correlação com o aparecimento da febre e/ou instabilidade térmica no recém-nascido. ${ }^{(7,26)}$

Portanto, no choque séptico não raramente ocorre coagulaçáo intravascular disseminada (CIVD), resultante da geração sustentada de trombina, que causa trombose micro-vascular com disfunção orgânica final, e paradoxalmente, diátese hemorrágica devido ao consumo de fatores da coagulação. $(27,28)$ Sistema imune e coagulação estão intimamente ligados. Citocinas que medeiam migraçáo e ativação de neutrófilos até os tecidos e no compartimento extravascular, geram o fator tecidual que inicia a formaçáo de trombina e depósito de fibrina. Trombina, por sua vez, estimula a ativaçáo de mais mediadores inflamatórios. A formação de fibrina estabiliza "plugs" plaquetários, além do importante papel na adesão dos patógenos na superfície dos leucócitos facilitando a fagocitose. . $^{(23,24,29)}$

Os receptores de membrana conhecidos como Toll-like receptors (TLRs) apresentam um papel fundamental na fisiopatologia do choque séptico, interferindo no sistema cardio- 
vascular, dependendo do patógeno que acionou a resposta inflamatória sistêmica. São receptores de membrana presentes nas células do sistema imune (células dendríticas, neutrófilos e monócitos) que conseguem detectar padróes moleculares associados à patógenos, conhecidos na literatura internacional como PAMPs. Exemplos de PAMPs são a endotoxina lipopolissacarídica (LPS) da membrana dos organismos gramnegativos e os ácidos teicóicos das bactérias gram-positivas. Através da ativação de moléculas sinalizadoras provocam no núcleo, em sítios de transcrição, a ativação do pacote apropriado de genes que induzirá mediadores pró-inflamatórios e antiinflamatórios, especialmente citocinas. ${ }^{(9,13,30)}$

Além das células do sistema imune, estes receptores podem ser expressos em outras células, como células endoteliais, células do epitélio alveolar e cardiomiócitos. A produção de TNF- $\alpha$ (fator de necrose tumoral alfa) e IL-1 $\beta$ (interleucina $1 \beta$ ) induzida por TLRs é responsabilizada pela disfunção miocárdica precoce na sepse grave por gram-negativos (TLR4) e gram-positivos (TLR2). ${ }^{(23,30)}$

Outra função dos TLRs é a amplificação da resposta inflamatória, pelo reconhecimento de padróes moleculares "perigo-associados" (DAMPs) liberados quando ocorre injúria celular. ${ }^{(23,30)}$

Os polimorfismos genéticos dos TLRs e proteínas sinalizadoras (MYD88) estáo relacionados com a intensidade da resposta inflamatória e a mortalidade no choque séptico. ${ }^{(30-32)}$ Cornell et al., recentemente, revisaram os complexos fatores genéticos, de expressão transcripcional e epigenéticos que regulam a resposta do hospedeiro a infecção e diferentes padrôes do choque séptico. ${ }^{(33)}$

\section{O papel do agente bacteriano}

Padróes hemodinâmicos distintos podem sugerir o patógeno no choque séptico em crianças, com predomínio de bactérias gram negativas no choque frio e gram positivas no choque quente. ${ }^{(34)}$ Não há paralelo descrito no período neonatal. Perfusão periférica aparentemente preservada (pulsos cheios e extremidades vasodilatadas) na presença de oligúria, acidemia, alteração de consciência e hipotensão, que indica a presença de vasoplegia ou choque quente, eventualmente estará presente no período de transição de prematuros extremos e na sepse tardia de prematuros pelo estafilococo, e pode produzir a impressão de falsa estabilidade. ${ }^{(1,2,34,35)}$

Os germes gram-positivos, como o Streptococcus do grupo $\mathrm{B}$, são os mais freqüentemente descritos como agentes etiológicos na sepse neonatal precoce. No entanto, mais recentemente alguns patógenos gram negativos têm sido muito descritos, principalmente na sepse cuja transmissão é vertical. ${ }^{(35,36)}$ Dados do CDC - Centers for Disease Control and Prevention apontam que o uso de antibiótico intraparto pode ter re- duzido a incidência de sepse por gram postivo, embora tenha facilitado o crescimento da flora bacteriana gram negativa. ${ }^{(37)}$

A doença estreptocócica invasiva está relacionada com ausência de anticorpos maternos anti-capsulares. Quando o recém-nascido aspira o patógeno presente no canal vaginal (colonização materna) poderá ocorrer invasão pulmonar, esta é dependente em grande parte do atraso na expressão gênica da proteína $\mathrm{A}$ do surfactante, que será tanto maior quanto menor a idade gestacional ao nascimento. Em seguida, a bactéria atinge a corrente sangüínea e promove intensa síndrome da resposta inflamatória sistêmica, classicamente descrita na sepse precoce. Já a cepa tipo III do estreptococo é responsável pela sepse neonatal tardia e geralmente está associada com meningite. ${ }^{(36,38)}$

$\mathrm{Na}$ sepse tardia, assim como em qualquer situação de hospitalização prolongada, o Staphylococcus aureus é germe mais freqüente. É um organismo gram-positivo encapsulado, cuja presença da proteína A na parede celular confere a capacidade de bloquear a opsonização e esta ação anti-fagocitose prejudica a resposta natural do hospedeiro, razão pela qual é freqüente a manifestação de bacteremia persistente e focos locais de difícil tratamento. A emergência de cepas multi-resistentes (MRSA), inclusive com resistência à vancomicina, é um problema grave nas unidades de terapia intensiva (UTIs) neonatais. ${ }^{(38)}$ Staphylococcus coagulase-negativo (SCON), e seus sub-tipos mais conhecidos; $\mathrm{S}$. epidermidis, $\mathrm{S}$ haemolyticus, S. capitis. S. warnier, são os agentes mais freqüentes na infecçấo nosocomial em prematuros de muito baixo peso. Agem pela indução de uma resposta inflamatória leve com ácidos lipoproteicos, peptideoglicanos e pró-albuminas. A apresentação é insidiosa, com apnéias mais freqüentes que o padrão habitual, piora do padrão ventilatório, letargia, instabilidade térmica e íleo. A capacidade de formar biofilme, uma camada extracelular de proteínas e polissárides que permite adesão a cateteres intravasculares, é o principal mecanismo patogênico. No entanto, a maior dificuldade ainda é estabelecer quando realmente é infecção invasiva e quando é contaminação. Não há um marcador suficentemente sensível e específico que possa fazer esta diferenciação. Parece que a maior capacidade de virulência relaciona-se ao tipo de distribuição de gens na formação do biofilme. ${ }^{(39,40)}$

A sepse fúngica é mais comum no prematuro extremo grave, em suporte de ventilação mecânica, com nutriçáo parenteral prolongada, hiperglicemia, e exposto a múltiplos esquemas de antibioticoterapia prévios. ${ }^{(41)}$ Candida albicans (vertical, pela flora materna) e candida parapsiloisis (horizontal, contaminaçáo nosocomial) estáo muito relacionados com presença de cateteres vasculares. $\mathrm{O}$ emprego de fluconazol profilático para prematuros extremos (peso de nascimento inferior a 1000 gramas e idade gestacional inferior a 
27 semanas) tem sido uma opção preventiva de mortalidade por choque séptico nesta população de alto risco. ${ }^{(42)} \mathrm{Na}$ UTI Neoantal do Hospital de Clínicas de Porto Alegre, estamos utilizando Anfotericina B empiricamente, baseando-se em fatores de risco restritos à população de pré-termos de muito baixo peso, e em detrimento do fluconazol profilático. Acreditamos que assim, selecionamos os casos potenciais e evitamos a super-resistência. ${ }^{(43,44)}$

\section{Particularidades do choque no recém-nascido}

Há diferenças marcantes entre choque no período neonatal e o choque no adulto ou na criança maior. O período de transiçáo pós-natal, adaptativo, do ponto de vista metabólico, celular e hemodinâmico, faz com que existam diferenças também entre o pré-termo e o recém-nascido de termo. ${ }^{(17,34)}$ A avaliação dos sinais vitais obedece a particularidades descritas no International Consensus Conference on Pediatric Sepsis e adaptadas no quadro 2 . $^{(16)}$

O prematuro e o recém-nascido sem maiores intercorrências, apresentam um estado de hipercoagulabilidade; onde se observa aumento dos receptores de trombomodulina no endotélio da microcirculação e diminuição da resposta a proteínas ligadas a anticoagulaçáo (proteína $\mathrm{C}$ ativada, entre outras). ${ }^{(24,28)}$

Nos casos mais graves, é acionada a via extrínseca que promove maior expressáo do fator tecidual no compartimento extravascular dos tecidos. Este parece ser o evento inicial nas alteraçôes da coagulação (CIVD); com ativação significativa do complemento e da cascata da coagulaçáo. Recentemente, foi demonstrado que antitrombina, fator de inativação tecidual e proteína $\mathrm{C}$ ativada estão diminuídos na sepse grave e a resposta fibrinolítica está alterada (ação do TNF- $\alpha$ ). O resultado é trombose microvascular, fibrinólise diminuída, aumento da permeabilidade capilar, e áreas hipoperfundidas ou incapazes de suprir o aumento da demanda metabólica dos tecidos irrigados levando a hipóxia tecidual e hipóxia citopática por dano mitocondrial. ${ }^{(45)}$

Os recém-nascidos e as crianças menores apresentam risco aumentado de sangramentos, em função dos baixos níveis circulantes de fatores pró-coagulantes vitamina K-dependentes (fatores II, VII, IX e X). O sistema de coagulação do recém-nascido tem todos os fatores de coagulação essenciais, porém em quantidade inferior ao adulto. A quantidade de plaquetas é semelhante entre os adultos e as crianças, mas nas crianças menores as plaquetas tendem a ser pouco responsivas a agonistas fisiológicos. ${ }^{(46,47)}$ Estas diferenças explicam, pelo menos parcialmente, os quadros mais graves de CIVD, maior mortalidade e pouca resposta terapêutica a moduladores da coagulação no recém-nascido, quando comparado com adultos e crianças. ${ }^{(1,45,48)}$

O sistema imune do recém-nascido apresenta uma reposta frequentemente dependente da imunidade inata que, por sua vez, é limitada em função quando comparada à imunidade de adultos e de crianças maiores. ${ }^{(1)}$ Há deficiência de componentes do complemento circulante, da expressáo de proteínas antimicrobianas e peptídios e das células T-helper. As funçóes dos neutrófilos, macrófagos, monócitos, células dendríticas e a resposta reduzida aos agonistas dos Toll-like receptors fazem do recém-nascido, especialmente o prematuro extremamente suscetível ao microorganismo invasor e ao choque séptico. ${ }^{(1,8,9)}$

O diagnóstico do choque séptico no recém-nascido é clínico e não se deve aguardar nenhum tipo de confirmação laboratorial para iniciar o manejo imediato. È necessário o conhecimento das faixas de normalidade dos sinais vitais, conforme faixa etária do recém-nascido já que não é disponível a medida direta do débito cardíaco, usualmente empregada em adultos. ${ }^{(34,49)}$ No recém-nascido a termo não é necessária presença de hipotensão para o diagnóstico de choque, mas hipotensão associada com sinais clínicos é choque séptico até prova em contrário. ${ }^{(10)}$ Os valores normais de pressão arterial no recém-nascido de muito baixo peso não são bem estabelecidos. Existem poucos artigos que exploram este tópico. Além disso, é muito difícil estabelecer valores normais numa população que necessita freqüentemente de ventilação mecânica, apresenta canal arterial patente e tem comumente alteraçôes hemodinâmicas. ${ }^{(50,51)}$

\section{O choque e a pressáo arterial sistêmica}

Em qualquer idade choque é uma alteração primária do fluxo sanguíneo e não da pressão arterial e, apesar da pressão arterial adequada ser vital para perfusão de órgãos vitais e

Quadro 2 - Sinais vitais em recém-nascidos e crianças

\begin{tabular}{|lcccc|}
\hline & $\begin{array}{c}\text { Frequência cardíaca } \\
(\mathrm{bpm})\end{array}$ & $\begin{array}{c}\text { Frequência respiratória } \\
(\mathrm{mov} / \mathrm{min})\end{array}$ & $\begin{array}{c}\text { Contagem leucócitos } \\
(103 / \mathrm{min} 3)\end{array}$ & $\begin{array}{c}\text { Pressão sistólica } \\
(\mathrm{mmHg})\end{array}$ \\
\hline $0-7$ dias & $<100 \mathrm{ou}>180$ & $>50$ & $>34$ ou $<5$ & $<65$ \\
$7-28$ dias & $<100 \mathrm{ou}>180$ & $>40$ & $>19,5$ ou $<5$ & $<75$ \\
$2-12$ meses & $<90 \mathrm{ou}>180$ & $>34$ & $>19,5$ ou $<5$ & $<100$ \\
\hline
\end{tabular}

Modificado de: International Consensus Conference on Pediatric Sepsis. International pediatric sepsis consensus conference: definitions for sepsis and organ dysfunction in pediatrics. ${ }^{(16)}$ 
Quadro 3 - Valores sugeridos de pressão arterial média para diagnóstico de hipotensão no recém-nascido

\begin{tabular}{|lllll|}
\hline Peso de nascimento & $<1000 \mathrm{~g}$ & $1000-1500 \mathrm{~g}$ & $1500-2500 \mathrm{~g}$ & $>2500 \mathrm{~g}$ \\
\hline Idade gestacional & $23-27$ semanas & $28-33$ semanas & $34-37$ semanas & $>37$ semanas \\
$1-3$ dias & PAM $<\mathrm{IG}$ & $<30$ & $<35$ & $<40$ \\
$4-7$ dias & $<30$ & $<33$ & $<35$ & $<45$ \\
$>7$ dias & $<30$ & $<35$ & $<40$ & $<50$ \\
\hline
\end{tabular}

Adapatada com permissão do II Consenso de Hemodinâmica SIBEN (Sociedade Ibero-Americana de Neonatologia); 2008. PAM - pressão arterial média; IG - idade gestacional.

para filtração glomerular, a ausência de hipotensão não exclui choque, pois a PA pode ser mantida a custa de mecanismos compensatórios. ${ }^{(51)}$ Esta afirmação adquire especial complexidade no período neonatal, onde a pressão arterial é variável com a idade, conforme amplamente discutido no Consenso de Hemodinâmica 2008 da Sociedade Ibero-Americana de Neonatologia (Quadro 3).

Nos prematuros extremos sem infecção, especialmente no período de transição, é observada pouca correlação entre PAM e fluxo sanguíneo sistêmico; estes quando "saudáveis" e com pressões sistêmicas menores que a média podem ter perfusão cerebral adequada, débito cardíaco normal e ausência de sinais clínicos ou bioquímicos de choque. Alguns autores sugerem hipotensão permissiva em pacientes saudáveis. ${ }^{(50-54)}$

No entanto, a hipotensão não é permissiva para os prematuros com choque séptico. Em prematuros criticamente enfermos, a hipotensão refratária está relacionada a canal arterial patente, hemorragia intraventricular e pior prognóstico. ${ }^{(8,48,53)}$ Por outro lado, a presença de canal arterial hemodinamicamente significativo parece influenciar deleteriamente a perfusão cerebral, o que traz sérias preocupaçóes quanto à reposição volêmica nesses pacientes. ${ }^{(5))}$ Técnicas de ultrassonografia para estimar fluxo em veia cava superior e débitos cardíacos são eficazes para substituir a PAM como instrumento de avaliação, mas não estão amplamente disponíveis. ${ }^{(10)}$

\section{Manejo volumétrico e farmacológico (vasopressores e inotrópicos)}

A atuação rápida para reversão precoce do choque séptico pediátrico-neonatal e a adoção das práticas voltadas para a recuperação hemodinâmica precoce com ressuscitação volumétrica e drogas vasoativas estáo associadas com bom prognóstico e diminuição significativa da mortalidade. ${ }^{(10,56)}$

È importante um pacote de medidas instituídas simultaneamente ao invés de medidas passo a passo, ou seja, o manejo é definido por tempo e metas. ${ }^{(10)} \mathrm{A}$ avaliação da microcirculação de prematuros sépticos revela que as alteraçôes já estão presentes 24 horas antes do aparecimento de parâmetros sistêmicos de sepse. ${ }^{(45)}$ Há a necessidade de mudarmos radicalmente a forma como pensamos o tempo no tratamento do choque séptico no neonato criticamente doente. Estudo recente observou que crianças menores de 2 anos e com doenças crônicas são especialmente sensíveis à pequena demora na ressuscitação volumétrica e são bastante responsivas a volume. ${ }^{(57)}$ Não há estudos semelhantes em recém-nascidos, mas é razoável que os mesmos princípios de tratamento se imponham ao período neonatal até melhor evidência.

Depois de assegurada a estabilidade da via aérea, a prioridade é a reposição volêmica com solução cristalóide (soro fisiológico 0,9\%); monitorização cuidadosa e especial atenção para a geração de descompensação hemodinâmica secundária ao canal arterial. ${ }^{(49,50)}$ Os limites da reposição volêmica da primeira hora são determinados basicamente pela idade gestacional; para o recém-nascido a termo é até $60 \mathrm{ml} / \mathrm{kg}$, em recém-nascidos pré-termo de muito baixo peso ( $\mathrm{PN}<$ 1500 gramas) até $30 \mathrm{ml} / \mathrm{kg}$ e nos prematuros extremos e sem evidências de perdas corporais significativas, até $20 \mathrm{ml} / \mathrm{kg}$. Respeitando-se estes limites da reposiçẫo volêmica, a solução salina é bem tolerada e não há descrição de efeitos adversos em recém-nascidos. ${ }^{(1,10,34)}$

A história de um prematuro extremo $(\mathrm{PN}<1000 \mathrm{~g})$ com antecedente de corioamnionite materna, que inicia nas primeiras 24 horas de vida com perfusão alterada, acidose metabólica, piora respiratória progressiva, taquicardia: presença de canal arterial silente (sem sopro) não é incomum nos primeiros três dias de vida. Neste caso, reposiçáo de volume excessiva ou rápida pode aumentar a intensidade do shunt e aumento da pré-carga em um miocárdio com pouca capacidade de estiramento e força contrátil. Falha em fechar o canal patente está associada com mortalidade aumentada, hemorragia peri-intraventricular e alteraçóes de neurodesenvolvimento. ${ }^{(58)}$ Volume em rápida infusão, acima de $30 \mathrm{ml} / \mathrm{kg}$ nas primeiras 48 horas de vida, foi associado com aumento da mortalidade. ${ }^{(59)}$

Reposição volumétrica promove hemodiluição, especialmente em recém-nascidos anêmicos ou prematuros com hemorragia intracraniana. No choque séptico, quando os níveis de hemoglobina são inferiores a $12 \mathrm{~g} / \mathrm{dL}(\mathrm{Hb}<12 \mathrm{~g} / \mathrm{dl})$, recomenda-se transfundir concentrado de hemácias. O emprego de solução de glicose 10\% como volume de manutenção é necessário, e a taxa de infusão desta glicose deve ser revista no caso de anormalidades eletrolíticas, do cálcio e da glicose. É 
importante evitar alteraçóes acentuadas dos níveis glicêmicos no choque séptico. ${ }^{(60,61)} \mathrm{A}$ toxicidade potencial da glicose na sepse grave é consenso, mas sua ação sobre a resposta imune, processo reparadores, formação de espécies reativas de oxigênio e alteraçóes do equilíbrio acido-básico não está completamente esclarecida. Picos de hiperglicemia parecem relacionados com a gravidade da doença, no entanto, os efeitos que a hiperglicemia sustentada ou a variação acentuada dos níveis glicêmicos representam ainda não estáo bem descritos. Em população pediátrica, níveis de glicose acima de $178 \mathrm{mg} / \mathrm{dL}$ aumentaram em três vezes a mortalidade. ${ }^{(61)}$

$\mathrm{O}$ controle da hiperglicemia com insulina em pré-termos com nutrição parenteral tem sido questionado, em função dos efeitos deletérios de episódios de hipoglicemia nestes recém-nascidos. ${ }^{\left({ }^{(62)}\right.}$ Aproximadamente $25 \%$ dos pacientes pediátricos criticamente doentes com insulina contínua desenvolvem hipoglicemia. ${ }^{(60)}$ Por outro lado, a insulina parece atuar inibindo a resposta inflamatória e aumentando a competência imune, em adultos. No recém-nascido, até melhores evidências, o uso de insulina está indicado apenas quando é reduzida a oferta de glicose e ainda assim, ocorre hiperglicemia acentuada $(>180 \mathrm{mg} / \mathrm{dl})$ e nos recém-nascidos com choque refratário e evolução desfavorável. (60,62) $^{(1)}$

A oferta de oxigênio aos tecidos hipóxicos é parte fundamental do tratamento, no entanto para os prematuros extremos, a oxigenação ótima no choque não está determinada. Se submetidos a controle muito restrito da saturação (entre 83\%-89\%), apresentam maior incidência de canal arterial patente. ${ }^{(63)}$ Por outro lado, efeitos deletérios da hiperóxia sobre a reperfusão de tecidos isquêmicos também são temidos. Em prematuros extremos, deve-se evitar saturação $>94 \%$. A PaO2 não deve ser mantida em níveis supra-sistêmicos. ${ }^{(50,52,58,63)}$

\section{Manejo farmacológico}

O manejo farmacológico do choque séptico inclui vasopressores, como: dopamina, noradrenalina e vasopressina, além de inotrópicos como: adrenalina, dobutamina e milrinona, que frequentemente são associados, visando a restauração da micro circulação e da perfusão tecidual. ${ }^{(10,34,64)}$

A maioria dos estudos a respeito da eficácia e segurança dos fármacos empregados no choque séptico, é em adultos, alguns em pacientes pediátricos, e muita pouca evidência científica no recém-nascido. Algumas publicações envolvendo ações destes fármacos no pré-termo e com base no recente Consenso do American College of Critical Care Medicine, poderemos resumir o manejo farmacológico no recém-nascido. ${ }^{(10,34,64)}$

Dopamina: é o vasopressor mais utilizado para tratamento do choque em neonatologia; precursor natural da adrenali- na e noradrenalina, estimula receptores dopaminérgicos, beta e alfa nesta ordem, na medida em que a dose é aumentada. No manejo inicial é prescrita dose de $5-10 \mathrm{mcg} / \mathrm{kg} / \mathrm{min}$, devendo ser ajustada em base individual, conforme os efeitos observados em cada recém-nascido. Geralmente os acréscimos são de $2,5 \mathrm{mcg} / \mathrm{kg} / \mathrm{min}$ a cada $10-15$ minutos. ${ }^{(65)}$ Como os depósitos de noradrenalina são imaturos no prematuro, pode haver resistência à sua ação, além de vasoconstrição pulmonar proporcional a vasoconstrição sistêmica. ${ }^{(10)}$ Valverde et al. observaram que prematuros com concentraçóes de 15 a 20 $10 \mathrm{mcg} / \mathrm{kg} / \mathrm{min}$ de dopamina apresentavam redução da liberação do TSH, dificultando o diagnóstico de hipotireoidismo e produzindo efeitos adversos importantes como taquicardia, arritmias, bradicardia, náuseas e vômitos. ${ }^{(66)}$

Noradrenalina: Uso limitado no choque neonatal, indicada no choque "quente" (doses de 0,05 a $0,5 \mathrm{mcg} / \mathrm{kg} / \mathrm{min}$ ), situaçáo incomum no recém-nascido. Em um estudo observacional (nível de evidência muito fraco), com 22 recémnascidos em choque séptico refratário a volume e à dopamina e dobutamina e com Hipertensão Pulmonar Persistente (HPPRN), Tourneux et al. utilizaram noradrenalina, constatando melhora na pressão sanguínea e nos demais dados hemodinâmicos relacionados à HPPRN. Posteriormente, estes autores descreveram o desempenho cardíaco e fluxo cerebral com o uso de noradrenalina nestes recém-nascidos, sugerindo efeitos positivos. ${ }^{(67)}$ Em adultos, onde noradrenalina é amplamente utilizada, há questionamentos se a melhora da pressão sanguínea da noradrenalina não ocorreria em função de um "encarceramento" micro-circulatório, por isso a necessidade de monitorizar a saturaçáo venosa mista de $\mathrm{O} 2$ com o seu uso. ${ }^{(10)}$

Vasopressina (e seu análogo sintético, terlipressina): é um hormônio que atua na homeostase dos líquidos, potente vasoconstritor. Indicada no choque com vasodilatação, mas seu uso em neonatologia é restrito apenas a relatos de casos. ${ }^{(10,68)}$ Pode causar isquemia coronária, mesentérica e cutânea, em altas doses. ${ }^{(64)}$ Estudo piloto, demonstrou que baixas doses de vasopressina é seguro (hiponatremia é comum) em crianças hemodinamicamente estáveis, mas em ventilação mecânica. ${ }^{(69)}$

Adrenalina: em doses baixas $(<0,03 \mathrm{mg} / \mathrm{kg} / \mathrm{min})$ é um potente inotrópico $(\beta 1)$, cronotrópico $(\alpha 1)$ e, pela estimulaçáo de receptores $\beta 2$ tem efeito vasodilatador sistêmico e pulmonar. Em doses maiores, possui efeito vasopressor $(\alpha$-adrenérgico) sobre as circulaçóes sistêmicas e pulmonares, aumentando pressão sistêmica mais do que a pressão pulmonar. Adrenalina melhora débito cardíaco, perfusão miocárdica e aumenta a resistência vascular mesentérica. Efeitos adversos da adrenalina incluem aumento da RVP levando a débito cardíaco e perfusão tecidual diminuídos, hipertensão, 
taquicardia e necrose por extravasamento de vasos periféricos. Em prematuros, aumento da glicose e do lactato são efeitos transitórios. ${ }^{(57)}$ Está indicada no choque refratário e resistente a volume, dopamina e dobutamina, na dose de 0,05 a 0,3 $\mathrm{mcg} / \mathrm{kg} / \mathrm{min}$. $^{(10)}$

Dobutamina: frequentemente associada à dopamina no choque séptico do recém-nascido; apresenta efeitos $\beta$-adrenérgicos, que tendem a vasodilatação e estimulação dos receptores miocárdicos $\alpha$-adrenérgicos, aumentando a contratilidade e freqüência cardíacas. Como diminui a pós-carga, é particularmente útil no choque séptico que se caracteriza por disfunção miocárdica e alta resistência periférica, apesar do efeito indefinido sobre melhora do fluxo coronário e da oferta de $\mathrm{O}_{2}$ ao miocárdio do recém-nascido. Nos prematuros, melhora o fluxo sanguíneo sistêmico avaliado por ecocardiografia, mas não é superior a dopamina para reversão da hipotensão no choque séptico. Dobutamina pode diminuir a resistência vascular pulmonar, tendo benefício adicional no tratamento da HPPRN. Deve-se ter cuidado para evitar o uso de dobutamina em pacientes com obstrução ao fluxo do VE. Pode provocar hipotensão se o paciente ainda estiver hipovolêmico e em altas doses provoca taquiarrtimias. ${ }^{(53)}$

No recém-nascido a termo ou próximo do termo, uma vez assegurada reposição de perdas anteriores e iniciada a ressuscitação volumétrica, está indicado dopamina em dose $\beta$-adrenérgica (inotrópica) de $5-9 \mathrm{mcg} / \mathrm{kg} / \mathrm{min}$ associada à dobutamina $2,5-10 \mathrm{mcg} / \mathrm{kg} / \mathrm{min}$, ainda na primeira hora do manejo. ${ }^{(10,65)}$ Já no choque do prematuro extremo no período de transição não está estabelecido qual é o melhor esquema inicial de drogas vasoativas. ${ }^{(10,57,58)}$ A medida mais importante é uniformizar a conduta no serviço para poder aferir resultados. O uso da dopamina é seguro nestes pacientes. ${ }^{(65)}$ Sugerimos o uso de esquema utilizando dopamina + dobutamina em doses inotrópicas como terapêutica inicial associada à reposição volumétrica mais cuidadosa naqueles prematuros extremos. ${ }^{(56)}$

Milrinona: inibidor seletivo da isoenzima fosfodiesterase com importante capacidade inotrópica, ao mesmo tempo em que provoca vasodilatação sistêmica e pulmonar (inodilatador). $\mathrm{O}$ uso bem-sucedido da milrinona no choque séptico pediátrico com alta resistência vascular periférica (choque "frio"), disfunção ventricular e pressão normal trouxe considerações sobre sua utilidade em recém-nascidos, especialmente na presença de HPPRN. Indicado no choque resistente a adrenalina, a dose preconizada é de $0,75 \mathrm{mcg} / \mathrm{kg} /$ min por 3 horas, seguida de $0,2 \mathrm{mcg} / \mathrm{kg} / \mathrm{min}$. É necessária reposição sistemática de volume além de doses vasopressoras de dopamina, estas podem ser utilizadas no choque refratário a adrenalina para contrabalancear o risco de hipotensão da milrinona. ${ }^{(10,64)}$ Paradisis e colaboradores não conseguiram demonstrar efeitos significativos da milrinona em prematuros extremos com baixo fluxo sistêmico nas primeiras 24 horas de vida, portanto, devendo ser utilizada em UTIs neonatais com condiçôes de controle hemodinâmico. ${ }^{(70)}$

\section{Outras possibilidades farmacológicas}

Corticosteróides: podem atuar no choque séptico melhorando a sensibilidade da parede do vaso a catecolaminas circulantes ou drogas vasoativas exógenas, inibindo a expressão da enzima óxido nítrico sintetase, ou pela supressão de respostas imunes. ${ }^{(1,10)}$ Além disso, recém-nascidos sépticos podem desenvolver insuficiência adrenal relativa, manifesta por baixos níveis de cortisol em situaçóes de stress. ${ }^{(71)}$

A terapia com hidrocortisona deve ser reservada para pacientes com choque refratário ou em serviços que não possuem estrutura para o uso de inodilatadores (milrinona); no choque resistente a adrenalina ou, ainda, na suspeita de insuficiência adrenal relativa ou absoluta (genitália ambígua). ${ }^{(56)}$

Agentes imunomoduladores: os agentes imunomodulares têm apresentado resultados frustrantes no manejo do choque séptico no recém-nascido. Imunoglobulina IV, fatores estimulantes de colônias (rhG-CSF and rhGM$\mathrm{CSF}$ ) proteína $\mathrm{C}$ ativada e pentoxifilina são agentes imunomodulares já aventados para uso no choque séptico neonatal. $^{(1,10,24)}$

A proteína C-ativada, potencialmente benéfica em pacientes adultos, não apresentou benefícios em crianças e está associada à hemorragia intracraniana em pacientes com menos de 60 dias de vida. ${ }^{(1)}$ Em modelo animal de recém-nascido foi observado melhora da microcirculação intestinal com rhAPC (proteína $\mathrm{C}$ recombinante humana, comercialmente disponível), após indução de choque endotóxico. ${ }^{(24)}$

Pentoxifilina, um anti-agregante plaquetário em prematuros, demonstrou reduzir mortalidade, comprometimento circulatório, coagulopatia e enterocolite necrosante quando comparado a placebo, e sem efeitos adversos conhecidos. No momento é uma opção promissora no choque refratário, na dose de $5 \mathrm{mg} / \mathrm{kg} /$ hora durante 6 horas, 5 dias sucessivos. ${ }^{(10)}$

\section{CONCLUSÁO}

O impacto do choque séptico neonatal nas taxas de morbi-mortalidade é elevado, mas algumas estratégias promissoras têm sido avaliadas, envolvendo o entendimento da fisiopatologia, dos determinantes genéticos e de imunomodulares na sepse neonatal, muitos dos quais, exclusividade do recémnascido. Protocolos institucionais práticos com medidas de controle de infecção hospitalar, de estabilização hemodinâmi$\mathrm{ca}$, e no futuro, imunomodulatórias devem ser incorporadas nas UTIs neonatais 
Com a finalidade de melhorar a qualidade da assistência neonatal, devem-se adotar medidas antecipatórias, prevenir, reconhecer precocemente, e tratar a sepse neonatal, revertendo rapidamente suas dramáticas conseqüências.

\section{ABSTRACT}

The nonspecific presentation of neonatal sepsis and systemic inflammatory response syndrome preceding septic shock delay the early diagnosis of septic shock and increase its mortality rate. Early diagnosis involves suspecting septic shock in every newborn with tachycardia, respiratory distress, difficult feeding, altered tonus and skin coloration, tachypnea and reduced perfusion, specially in case of maternal peripartum infection, cho- rioamnionitis or long-term membranes rupture.

This article aims to review current knowledge on neonatal period peculiarities, fetal circulation dynamics, and the pregnancy age variable.

Newborn septic shock is not just a small adult shock. In the newborn, the septic shock is predominantly cold and characterized by reduced cardiac output and increased systemic vascular resistance (vasoconstriction).

Time is fundamental for septic shock reversion. The indexed-databases literature review provides subside for the newborn management.

Keywords: Sepsis; Infant, newborn; Shock, septic; Cytokines; Systemic inflammatory response syndrome; Neonatal mortality (Public Health)

\section{REFERÊNCIAS}

1. Wynn J, Cornell TT, Wong HR, Shanley TP, Wheeler DS. The host response to sepsis and developmental impact. Pediatrics. 2010;125(5):1031-41.

2. Lawn JE, Cousens S, Zupan J, Lancet Neonatal Survival Steering Team. 4 million neonatal deaths: when? Where? Why? Lancet. 2005;365(9462):891-900.

3. Paananen R, Husa AK, Vuolteenaho R, Herva R, Kaukola T, Hallman M. Blood cytokines during the perinatal period in very preterm infants: relationship of inflammatory response and bronchopulmonary dysplasia. J Pediatr. 2009;154(1):39-43.e3.

4. Polglase GR, Hillman NH, Pillow JJ, Cheah FC, Nitsos I, Moss TJ, et al. Positive end-expiratory pressure and tidal volume during initial ventilation of preterm lambs. Pediatr Res. 2008;64(5):517-22.

5. Yoon BH, Romero R, Park JS, Kim JC, Kim SH, Choi JH, Han TR. Fetal exposure to an intra-amniotic inflammation and the development of cerebral palsy at the age of three years. Am J Obstet Gynecol. 2000;182(3):675-81.

6. Kadhim H, Tabarki B, Verellen G, De Prez C, Rona AM, Sébire G. Inflamatory cytokines in the pathogenesis of periventricular leukomalacia. Neurology. 2001;56(10):1278-84.

7. Silveira RC, Procianoy RS, Dill JC, da Costa CS. Periventricular leukomalacia in very low birth weight preterm neonates with high risk for neonatal sepsis. J Pediatr (Rio J). 2008;84(3):211-6.

8. Adams-Chapman I, Stoll BJ. Neonatal infection and longterm neurodevelopmental outcome in the preterm infant. Curr Opin Infect Dis. 2006;19(3):290-7. Review.

9. Levy O. Innate immunity of the newborn: basic mechanisms and clinical correlates. Nat Rev Immunol. 2007;7(5):379-90.

10. Brierley J, Carcillo JA, Choong K, Cornell T, Decaen
A, Deymann A, et al. Clinical practice parameters for hemodynamic support of pediatric and neonatal septic shock: 2007 update from the American College of Critical Care Medicine. Crit Care Med. 2009;37(2):666-88. Erratum in: Crit Care Med. 2009;37(4):1536. Skache, Sara [corrected to Kache, Saraswati]; Irazusta, Jose [corrected to Irazuzta, Jose].

11. Yafeng Dong, Weijian Hou, Jiaxue Wei, Weiner CP. Chronic hypoxemia absent bacterial infection is one cause of the fetal inflammatory response syndrome (FIRS). Reprod Sci. 2009;16(7):650-6.

12. Silveira RC, Procianoy RS. Níveis plasmáticos de interleucina-1 e interleucina-6 em recém-nascidos com febre. J Pediatr (Rio J). 1999;75(1):29-33.

13. Munford RS, Pugin J. Normal responses to injury prevent systemic inflammation and can be immunosuppressive. Am J Respir Crit Care Med. 2001;163(2):316-21.14. S Silveira RC, Procianoy RS. Evaluation of interleukin-6, tumor necrosis factor-alpha and interleukin-1beta for early diagnosis of neonatal sepsis. Acta Paediatr. 1999;88(6):647-50.

15. Meadow W, Rudinsky B. Inflammatory mediators and neonatal sepsis. Rarely has so little been known by so many about so much. Clin Perinatol. 1995;22(2):519-36.

16. Goldstein B, Giroir B, Randolph A; International Consensus Conference on Pediatric Sepsis. International pediatric sepsis consensus conference: definitions for sepsis and organ dysfunction in pediatrics. Pediatr Crit Care Med. 2005;6(1):2-8. Review.

17. Brilli RJ, Goldstein B. Pediatric sepsis definitions: past, present, and future. Pediatr Crit Care Med. 2005;6(3 Suppl):S6-8.

18. Fernandes RM, van der Lee JH, Offringa M. A systematic review of the reporting of Data Monitoring Committees roles, interim analysis and early termination in pediatric clinical trials. BMC Pediatr. 2009; 9:77.

19. Short MA. Linking the sepsis triad of inflammation, 
coagulation, and suppressed fibrinolysis to infants. Adv Neonatal Care. 2004;4(5):258-73.

20. Procianoy RS, Silveira RC. The role of sample collection timing on interleukin-6 levels in early-onset neonatal sepsis. J Pediatr (Rio J). 2004;80(5):407-10.

21. Kurt AN, Aygun AD, Godekmerdan A, Kurt A, Dogan Y, Yilmaz E. Serum IL-1beta, IL-6, IL-8, and TNF-alpha levels in early diagnosis and management of neonatal sepsis. Mediators Inflamm. 2007;2007:31397.

22. Berner R, Niemeyer CM, Leititis JU, Funke A, Schwab $\mathrm{C}$, Rau U, et al. Plasma levels and gene expression of granulocyte colony-stimulating factor, tumor necrosis factor-alpha, interleukin (IL)-1beta, IL-6, IL-8, and soluble intercellular adhesion molecule- 1 in neonatal early onset sepsis. Pediatr Res. 1998;44(4):469-77.

23. Fleer A, Krediet TG. Innate immunity: toll-like receptors and some more. A brief history, basic organization and relevance for the human newborn. Neonatology. 2007;92(3):145-57.

24. Fischer D, Nold MF, Nold-Petry CA, Furlan A, Veldman A. Protein $C$ preserves microcirculation in a model of neonatal septic shock. Vasc Health Risk Manag. 2009;5:775-81.

25. Cinel I, Opal SM. Molecular biology of inflammation and sepsis: a primer. Crit Care Med. 2009;37(1):291-304.

26. Anderson MR, Blumer JL. Advances in the therapy for sepsis in children. Pediatr Clin North Am. 1997;44(1):179205. Review.

27. Kenet G, Strauss T, Kaplinsky C, Paret G. Hemostasis and thrombosis in critically ill children. Semin Thromb Hemost. 2008;34(5):451-8.

28. Levi M. The coagulant response in sepsis and inflammation. Hamostaseologie. 2010;30(1):10-2, 14-6. Review.

29. Levi M, van der Poll T. Inflammation and coagulation. Crit Care Med. 2010;38(2 Suppl):S26-34. Review.

30. Gao H, Leaver SK, Burke-Gaffney A, Finney SJ. Severe sepsis and Toll-like receptors. Semin Immunopathol. 2008;30(1):29-40. Review.

31. Leaver SK, Finney SJ, Burke-Gaffney A, Evans TW. Sepsis since the discovery of Toll-like receptors: disease concepts and therapeutic opportunities. Crit Care Med. 2007;35(5):1404-10.

32. Arcaroli J, Fessler MB, Abraham E. Genetic polymorphisms and sepsis. Shock. 2005;24(4):300-12. Review.

33. Cornell TT, Wynn J, Shanley TP, Wheeler DS, Wong HR. Mechanisms and regulation of the gene-expression response to sepsis. Pediatrics. 2010;125(6):1248-58.

34. Brierley J, Peters MJ. Distinct hemodynamic patterns of septic shock at presentation to pediatric intensive care. Pediatrics. 2008;122(4):752-9.

35. Stoll BJ, Hansen N, Fanaroff AA, Wright LL, Carlo WA, Ehrenkranz RA, et al. Changes in pathogens causing earlyonset sepsis in very-low-birth-weight infants. $\mathrm{N}$ Engl J Med. 2002;347(4):240-7.

36. Stoll BJ, Hansen NI, Higgins RD, Fanaroff AA, Duara S,
Goldberg R, Laptook A, Walsh M, Oh W, Hale E; National Institute of Child Health and Human Development. Very low birth weight preterm infants with early onset neonatal sepsis: the predominance of gram-negative infections continues in the National Institute of Child Health and Human Development Neonatal Research Network, 20022003. Pediatr Infect Dis J. 2005;24(7):635-9.

37. Schrag SJ, Stoll BJ. Early-onset neonatal sepsis in the era of widespread intrapartum chemoprophylaxis. Pediatr Infect Dis J. 2006;25(10):939-40. Review.

38. 38. Stoll BJ, Hansen N, Fanaroff AA, Wright LL, Carlo WA, Ehrenkranz RA, et al. Late-onset sepsis in very low birth weight neonates: the experience of the NICHD Neonatal Research Network. Pediatrics. 2002;110(2 Pt 1):285-91.

39. Bradford R, Abdul Manan R, Daley AJ, Pearce C, Ramalingam A, D'Mello D, et al. Coagulase-negative staphylococci in very-low-birth-weight infants: inability of genetic markers to distinguish invasive strains from blood culture contaminants. Eur J Clin Microbiol Infect Dis. 2006;25(5):283-90.

40. Klingenberg C, Rønnestad A, Anderson AS, Abrahamsen TG, Zorman J, Villaruz A, et al. Persistent strains of coagulase-negative staphylococci in a neonatal intensive care unit: virulence factors and invasiveness. Clin Microbiol Infect. 2007;13(11):1100-11.

41. van den Hoogen A, Gerards LJ, Verboon-Maciolek MA, Fleer A, Krediet TG. Long-term trends in the epidemiology of neonatal sepsis and antibiotic susceptibility of causative agents. Neonatology. 2010;97(1):22-8.

42. Kaufman DA. Challenging issues in neonatal candidiasis. Curr Med Res Opin. 2010;26(7):1769-78.

43. Procianoy RS, Silveira RC. Prophylactic fluconazole in preterm neonates. N Engl J Med. 2007;357(13):1349; author reply 1349.

44. Procianoy RS, Enéas MV, Silveira RC. Empiric guidelines for treatment of Candida infection in high-risk neonates. Eur J Pediatr. 2006;165(6):422-3.

45. 45. Weidlich K, Kroth J, Nussbaum C, Hiedl S, Bauer A, Christ F, Genzel-Boroviczeny O. Changes in microcirculation as early markers for infection in preterm infants-- an observational prospective study. Pediatr Res. 2009;66(4):461-5.

46. Israels SJ, Rand ML, Michelson AD. Neonatal platelet function. Semin Thromb Hemost. 2003;29(4):363-72. Review.

47. Kuhle S, Male C, Mitchell L. Developmental hemostasis: pro-and anticoagulant systems during childhood. Semin Thromb Hemost. 2003;29(4):329-38.

48. Stoll BJ, Hansen NI, Adams-Chapman I, Fanaroff AA, Hintz SR, Vohr B, Higgins RD; National Institute of Child Health and Human Development Neonatal Research Network. Neurodevelopmental and growth impairment among extremely low-birth-weight infants with neonatal 
infection. JAMA. 2004;292(19):2357-65.

49. Goldstein B. Heart rate characteristics in neonatal sepsis: a promising test that is still premature. Pediatrics. 2005;115(4):1070-2.

50. Hamrick SE, Hansmann G. Patent ductus arteriosus of the preterm infant. Pediatrics. 2010;125(5):1020-30.

51. Cayabyab R, McLean CW, Seri I. Definition of hypotension and assessment of hemodynamics in the preterm neonate. J Perinatol. 2009;29 Suppl 2:S58-62.

52. Weindling AM, Subhedar NV. The definition of hypotension in very low-birthweight infants during the immediate neonatal period. NeoReviews. 2007;8(1):e32-43.

53. Dempsey EM, Barrington KJ. Evaluation and treatment of hypotension in the preterm infant. Clin Perinatol. 2009; 36(1):75-85. Review.

54. Munro MJ, Walker AM, Barfield CP. Hypotensive extremely low birth weight infants have reduced cerebral blood flow. Pediatrics. 2004;114(6):1591-6. Erratum in: Pediatrics. 2005;115(6):1794-5.

55. Sarkar S, Dechert R, Schumacher RE, Donn SM. Is refractory hypotension in preterm infants a manifestation of early ductal shunting? J Perinatol. 2007;27(6):353-8.

56. Vincent JL. Clinical sepsis and septic shock--definition, diagnosis and management principles. Langenbecks Arch Surg. 2008;393(6):817-24. Review.

57. Oliveira CF, Nogueira de Sá FR, Oliveira DS, Gottschald AF, Moura JD, Shibata AR, et al. Time- and fluid-sensitive resuscitation for hemodynamic support of children in septic shock: barriers to the implementation of the American College of Critical Care Medicine/Pediatric Advanced Life Support Guidelines in a pediatric intensive care unit in a developing world. Pediatr Emerg Care. 2008;24(12):810-5.

58. Noori S, McCoy M, Friedlich P, Bright B, Gottipati V, Seri I, Sekar K. Failure of ductus arteriosus closure is associated with increased mortality in preterm infants. Pediatrics. 2009;123(1):e138-44.

59. Ewer AK, Tyler W, Francis A, Drinkall D, Gardosi JO. Excessive volume expansion and neonatal death in preterm infants born at 27-28 weeks gestation. Paediatr Perinat Epidemiol. 2003;17(2):180-6.

60. Vlasselaers D, Milants I, Desmet L, Wouters PJ, Vanhorebeek I, van den Heuvel I, et al. Intensive insulin therapy for patients in paediatric intensive care: a prospective, randomised controlled study. Lancet. 2009;373(9663):547-56.

61. Branco RG, Garcia PC, Piva JP, Casartelli CH, Seibel V, Tasker RC. Glucose level and risk of mortality in pediatric septic shock. Pediatr Crit Care Med. 2005;6(4):470-2.

62. Beardsall K, Vanhaesebrouck S, Ogilvy-Stuart AL, Vanhole C, Palmer CR, van Weissenbruch M, et al. Early insulin therapy in very-low-birth-weight infants. N Engl J Med. 2008;359(18):1873-84.

63. Noori S, Patel D, Friedlich P, Siassi B, Seri I, Ramanathan R. Effects of low oxygen saturation limits on the ductus arteriosus in extremely low birth weight infants. J Perinatol. 2009;29(8):553-7.

64. Irazuzta J, Sullivan KJ, Garcia PC, Piva JP. Pharmacologic support of infants and children in septic shock. J Pediatr (Rio J). 2007;83(2 Suppl):S36-45. Review.

65. Pellicer A, Bravo MC, Madero R, Salas S, Quero J, Cabañas F. Early systemic hypotension and vasopressor support in low birth weight infants: impact on neurodevelopment. Pediatrics. 2009;123(5):1369-7666. Valverde E, Pellicer A, Madero R, Elorza D, Quero J, Cabañas F. Dopamine versus epinephrine for cardiovascular support in low birth weight infants: analysis of systemic effects and neonatal clinical outcomes. Pediatrics. 2006;117(6):e1213-22.

67. Tourneux P, Rakza T, Abazine A, Krim G, Storme L. Noradrenaline for management of septic shock refractory to fluid loading and dopamine or dobutamine in full-term newborn infants. Acta Paediatr. 2008;97(2):177-80.

68. Baldasso E, Ramos Garcia PC, Piva JP, Einloft PR. Hemodynamic and metabolic effects of vasopressin infusion in children with shock. J Pediatr (Rio J). 2007;83(5 Suppl):S137-45.

69. Baldasso E, Garcia PC, Piva JP, Branco RG, Tasker RC. Pilot safety study of low-dose vasopressin in nonseptic critically ill children. Intensive Care Med. 2009;35(2):355-9.

70. Paradisis M, Evans N, Kluckow M, Osborn D. Randomized trial of milrinone versus placebo for prevention of low systemic blood flow in very preterm infants. J Pediatr. 2009;154(2):189-95.

71. Fernandez EF, Montman R, Watterberg KL. ACTH and cortisol response to critical illness in term and late preterm newborns. J Perinatol. 2008;28(12):797-802. 